\title{
Caracterización de las Propiedades Mecánicas de dos Variedades de Batata (Ipomoea batatas Lam)
}

\author{
Armando Alvis*, Jaidith Jiménez y Guillermo Arrazola \\ Universidad de Córdoba, Programa de Ingeniería de Alimentos, Facultad de Ciencias Agrícolas, \\ Grupo de Investigación Procesos y Agroindustria de vegetales, Carrera 6 № 76-103, Km 3, vía Cereté, \\ Córdoba-Colombia. (e-mail: aalvis2@hotmail.com)
}

${ }^{*}$ Autor a quien debe dirigirse la correspondencia.

Recibido Dic. 26, 2014; Aceptado Feb. 11, 2015; Versión final Feb. 23, 2015, Publicado Ago. 2015

\section{Resumen}

En el presente trabajo se realizó un estudio de algunas propiedades mecánicas de dos variedades de batata (amarilla y roja). Esto con el objetivo de obtener datos necesarios para ser utilizados en cálculos de ingeniería de procesos, y en manipulación y almacenamiento del producto. Se utilizaron diferentes pruebas (penetración, compresión y flexión), para calcular los parámetros de esfuerzo máximo de penetración, esfuerzo y deformación en la fractura, y módulo de deformabilidad. Se utilizó el analizador de textura TA.XT plus Stable Micro Systems Se concluye que la variedad roja y amarilla presentan valores de esfuerzo de compresión muy similares $(0.77$ y $0.75 \mathrm{MPa})$ y que la deformación a que debe someterse para provocar la fractura son también muy parecidas (21.5 y $20.5 \%)$. En cuanto al esfuerzo de fractura se observaron diferencias significativas entre las dos variedades.

Palabras clave: batata, Ipomoea batatas Lam., propiedades mecánicas, esfuerzo, compresión y flexión

\section{Characterization of Mechanical Properties of Two Varieties of Sweet Potato (Ipomoea batatas Lam.)}

\begin{abstract}
In this paper a study of some mechanical properties of two varieties of sweet potato (yellow and red) is presented. This with the aim of obtaining data required in process engineering calculations, and in handling and storage of the product. Different tests were used (penetration, compression and bending), to estimate parameters of maximum penetration stress, stress and strain at fracture and modulus of deformability. The texture analyzer TA.XT plus Stable Micro Systems was used. It is concluded that the red and yellow variety have very similar values of compression stress $(0.77$ and $0.75 \mathrm{MPa})$ and that the deformation that must be applied to cause fracture are also very similar (21.5 and 20.5\%). With respect to the fracture stress, significant differences between the two varieties were observed.
\end{abstract}

Keywords: sweet potato, Ipomoea batatas Lam., mechanical properties, stress, compression and bending 


\section{INTRODUCCIÓN}

La batata (Ipomoea batatas Lam) es una de las hortalizas de más antigua domesticación, se cultiva ampliamente en muchos países, especialmente en China y en los países del Sudeste Asiático (An et al., 2003). Con el creciente interés del público por consumir productos naturales beneficiosos para la salud, la batata resulta un alimento por demás atractivo (Martí et al., 2011). Combina características de los cereales, en cuanto a su contenido de energía, y de otras frutas y hortalizas, por su contenido en fibra, vitaminas, minerales y otros compuestos benéficos para la salud como los antioxidantes (Martí, 2012). Este tubérculo, está compuesto de $60-80 \%$ de agua y $10-30 \%$ de almidón (Zhang et al., 2002), también contiene una variedad de nutrientes, como fibra, carotenoides, vitaminas, lisina y elementos minerales, entre otros, por lo que la batata es utilizada ampliamente en la industria alimentaria, industria química. Estudios recientes muestran que la batata tiene propiedades antioxidante (Teow et al., 2007; Tian y Wang, 2008)

En la agricultura moderna y en el procesamiento de alimentos, se utiliza cada vez más técnicas y equipos mecánicos, térmicos y otros. A pesar de ello, poco se conoce acerca de las propiedades físicas del material que se procesa y que indiscutiblemente influyen en la eficiencia de la maquinaria u operación así como en la calidad del producto final. Debido a esto, el conocimiento acerca de las propiedades físicas y entre ellas, las propiedades mecánicas de los productos agrícolas, no solo constituye un aspecto de gran importancia para ingenieros sino para tecnólogos, productores y otros campos de las ciencias como las agrícolas, que pudieran permitir la explotación de estas propiedades básicas y encontrar nuevas aplicaciones y usos para dichos productos (Mohsenin, 1965; Alvis et al., 2009). Además, a través de las mediciones reológicas pueden revelarse los cambios microestructurales debidos a daños mecánicos que sufren estos productos, tal como señalan Varela et al. (2007) para el caso de dos variedades diferentes de manzana y Alvis et al. (2009) dos variedades de ñame. En la medición de las propiedades mecánicas se emplean comúnmente diversas técnicas de ensayo, así por ejemplo, las propiedades elásticas de frutas y vegetales pueden ser estimadas a través de una simple prueba de penetración acoplada a un texturómetro donde se obtienen las curvas de fuerza versus distancia para calcular parámetros mecánicos tales como: fuerza o esfuerzo de penetración (Voisey et al., 1969; Anzaldúa et al, 1992; Moreira et al., 1999; Álvarez et al., 2000; Alvis et al., 2009).

La textura es uno de los principales atributos de calidad utilizados en la industria de alimentos frescos y procesados y para evaluar la calidad del producto y la aceptabilidad; los atributos de textura también se utilizan a lo largo de la cadena de valor de los alimentos para monitorear y controlar la calidad, que van desde la recolección para vegetales, para evaluar los impactos de manejo poscosecha y operación de procesamiento sobre la vida útil del producto y la referencia del consumidor y aceptabilidad. La manipulación y las condiciones de manejo como la temperatura de almacenamiento por lo general tienen efectos distintos sobre las propiedades mecánicas y de textura de los alimentos (Konopacka y Płocharski, 2004; Lana et al., 2005; Farag et al., 2009). Durante el procesamiento y conservación de los alimentos se dan cambios deseables y no deseables en la textura (Foegeding et al., 2010; Saeleaw y Schleining, 2011b; Morris y Morris, 2012).

Las pruebas de compresión uniaxial han sido también ampliamente utilizadas. En estos casos se obtienen los datos de esfuerzo vs. deformación y se pueden calcular el esfuerzo máximo de ruptura, el módulo de deformabilidad, energía de ruptura y otros parámetros (Pitt, 1984; Álvarez y Canet, 1998; Alvis et al., 2009). La prueba de flexión o doblado puede resultar de gran interés para evaluar el esfuerzo de fractura (Bruns y Bourne, 1975; Alvis et al., 2009) o la flexibilidad de determinados tejidos de frutas y vegetales (Lima y Singh, 2001) y a su vez, relacionarlo con la frescura o crocancia de productos tales como: zanahoria, pepino, papa, ñame (De Hombre et al., 2001; Alvis et al., 2009).En el presente trabajo se realizó un estudio para caracterizar desde el punto de vista reológico, las principales propiedades mecánicas de dos variedades de batata roja y amarilla, frescos cultivadas en la Región Atlántica de Colombia con el objetivo de obtener los datos necesarios para determinados cálculos de ingeniería de procesos, manipulación y almacenamiento del producto.

\section{MATERIALES Y METODOS}

Se seleccionaron dos variedades de batatas, amarillas y coloradas; provenientes del Banco de Germoplasma de la Universidad de Córdoba. Se recolectaron 30 kilogramos de muestra con tamaños y pesos semejantes. Las batatas se conservaron enteras a temperatura y humedad ambiente durante el desarrollo de la investigación.

Para la prueba de penetración, se empleó un vástago cilíndrico inoxidable de $2 \mathrm{~mm}$ de diámetro, acoplado al analizador de textura TA.XT plus Stable Micro Systems, para calcular el esfuerzo máximo de penetración; los ensayos se realizaron en el tubérculo entero con cascara, midiendo inicialmente con una cinta métrica 
desde el extremos distal hasta el extremo proximal, se marcaron los puntos 25,50 y $75 \%$ de la longitud obtenida, y en estos se aplicó la fuerza de penetración a las dos variedades de batata, hasta una distancia de penetración de $20 \mathrm{~mm}$. Para la prueba de compresión uniaxial la muestra se adecuó en forma de un cubo de $2 \mathrm{~cm}$ de aristas. La compresión se llevó a cabo hasta la ruptura y de esta forma se estimó el esfuerzo y deformación de fractura así como el módulo de deformidad. Se realizó una compresión uniaxial hasta $30 \%$ de la altura de la muestra. La Prueba de flexión se realizó, utilizando un puente de flexión instalado en el mismo equipo y fue utilizado para calcular el esfuerzo de fractura según (Bruns y Bourne, 1975). Para ello la muestra se cortó en forma de paralelepípedo de sección transversal cuadrada $(0.8 \times 0.8$ $\mathrm{cm}$ ) y longitud de $4 \mathrm{~cm}$. La muestra se colocó sobre el puente a una distancia entre los soportes de $2 \mathrm{~cm}$ y se aplicó una fuerza en el centro hasta lograr la fractura.

En todos los casos anteriores, las pruebas se realizaron a una velocidad de deformación de $5 \mathrm{~cm} / \mathrm{min}$ a temperatura ambiente $\left(25^{\circ} \mathrm{C}\right)$, tomando 3 réplicas de cada experimento.Se utilizó una prueba de t-student para establecer si existen diferencias entre los valores medidos del esfuerzo máximo de penetración entre las dos variedades de estudio.

\section{RESULTADOS Y DISCUSIÓN}

En la Tabla 1, se muestran los resultados del esfuerzo máximo de penetración correspondientes a las dos variedades de batata en las diferentes posiciones de 25, 50 y $75 \%$ de la longitud. Se puede observar de los resultados de la prueba de t- Student, los valores del esfuerzo máximo de penetración, en las tres posiciones y vemos que en el punto de penetración de $50 \%$ de la longitud no presenta diferencias significativas mientras que para el caso de los otros dos puntos de penetración 25 y $75 \%$ de la longitud existen diferencias significativas entre las variedades de estudio. Las diferencias entre variedades se puede deber tanto a la estructura como a la composición del producto (Mohsenin, 1965, Ciro et al., 2005; Alvis et al., 2009). Para la posición transversal la resistencia a la penetración varía según la posición de muestreo: (25 y $75 \%$ ) presentan la mayor y menor condición de resistencia del producto respectivamente. resultados similares obtuvo Buitrago et al. (2004) en las pruebas de compresión, de papa, pudiéndose observar que la deformación presenta una variación que oscila entre el $11 \%$ y $24 \%$.

Tabla 1. Resultados del esfuerzo máximo de penetración ( $\sigma$ máx.) expresado en MPa

\begin{tabular}{|c|c|c|c|}
\hline & \multicolumn{3}{|c|}{ Posiciones } \\
\hline Variedad & $25 \%$ & $50 \%$ & $75 \%$ \\
\hline Batata amarilla & $8.450 \pm 0.98$ & $7.272 \pm 0.68$ & $6.897 \pm 0.25$ \\
\hline Batata colorada & $7.439 \pm 0.93$ & $7.064 \pm 0.48$ & $5.122 \pm 0.39$ \\
\hline Prueba (t- Stu) & Sign. & N.Sign & Sign \\
\hline
\end{tabular}

En la Tabla 2 se muestran los resultados encontrados de la prueba de compresión uniaxial en el momento de fractura del cubo de $2 \mathrm{~cm}$ de arista obtenidos de la masa del producto.

Tabla 2. Resultados de la prueba de compresión uniaxial hasta fractura.

\begin{tabular}{|l|c|c|c|}
\hline \multicolumn{1}{|c|}{ Variedad } & Esfuerzo $(\mathrm{MPa})$ & Deformación $(\mathrm{mm})$ & Módulo de deformabilidad $(\mathrm{MPa})$ \\
\hline Batata amarilla & $0.772 \pm 0.05$ & $0.215 \pm 0.01$ & $3.599 \pm 0.24$ \\
\hline Batata colorada & $0.753 \pm 0.04$ & $0.176 \pm 0.02$ & $4.394 \pm 0.87$ \\
\hline Prueba $(\mathrm{t}$-Stu) & N.Sign & Sign. & N.Sign \\
\hline
\end{tabular}

Esta última prueba ratifica los resultados obtenidos hasta el momento, que la variedad amarilla es un poco más resistente a los esfuerzos aplicados y por consiguiente, menos susceptible a los daños mecánicos que puedan producirse durante la cosecha, manipulación, almacenamiento y procesamiento, factores que han sido considerados por (Nwandikom, 1990) como aquellos que pueden limitar la mecanización de la producción y por lo tanto su productividad. Igualmente Qian et al. (2010) obtuvieron para el esfuerzo aplicado a una muestra de batata valores de $0.92 \pm 0.23 \mathrm{MPa}$, siendo similares a los obtenidos en esta investigación, Alvis et al. (2009) encontraron valores en el esfuerzo de 0,31 MPa para ñame Diamante 22 y 0,57 para ñame Pico de Botella, muy por debajo a los obtenidos en este trabajo, en el módulo de deformabilidad los valores encontrados en esta investigación están por encima de los reportados en ñame variedad Diamante 22 y Pico de botella 1,4 y $1,7 \mathrm{MPa}$.

En la variedad amarilla notamos, que la fractura ocurre a valores un tanto superiores del esfuerzo, comparando con el comportamiento de la variedad colorada, lo cual permite que esta variedad pueda ser 
comprimida hasta deformaciones superiores al $20 \%$, en tanto que la variedad colorada se fractura a grados de compresión alrededor del $17 \%$. Para ñame Alvis et al. (2009) encontraron que en la variedad Pico Botella la fractura sucede a valores superiores al $30 \%$ en comparación con la variedad Diamante 22 que ocurre al $20 \%$ valor muy parecido a la Batata amarilla y colorada.. En la Tabla 3, se muestran los resultados de la fuerza de fractura y el grado de deformación correspondiente al momento de la fractura de la muestra, que se obtuvieron en la prueba de flexión para ambas variedades de batata.

Tabla 3. Resultados de la fuerza de fractura y la deformabilidad en flexión

\begin{tabular}{|c|c|c|}
\hline Variedad & Fuerza de fractura $(\mathrm{N})$ & Deformabilidad $(\mathrm{mm})$ \\
\hline Batata amarilla & $41.735 \pm 1.33$ & $6.3 \pm 0.4$ \\
\hline Batata colorada & $41.047 \pm 1.57$ & $5.9 \pm 0.3$ \\
\hline Prueba (t- Stu) & N.Sign & N.Sign \\
\hline
\end{tabular}

La ecuación 1, fue necesaria para calcular el esfuerzo de fractura, para una muestra de sección transversal rectangular colocada en un puente de flexión (Bruns y Bourne, 1975).

$\sigma=\frac{3 \mathrm{FL}}{2 \mathrm{bh}^{2}}$

En esta ecuación, b y h son las dimensiones del rectángulo, $L$ es la longitud del puente y $F$ es la fuerza de fractura. Reemplazando en la expresión anterior obtenemos que para la variedad colorada, el esfuerzo de fractura es de $1212.17 \mathrm{kPa}$, mientras que para la variedad amarilla es de $1262.85 \mathrm{kPa}$. Esto indica que la variedad amarilla soporta esfuerzos mecánicos mayores antes de su fractura que la variedad colorada y que además es algo más flexible ya que alcanza valores superiores de la deformación hasta el momento de la fractura tal como se evidencia en la Tabla 2, valores inferiores encontraron Ciro et al. (2005) en banano variedad Cavendish Valery de $125.58 \mathrm{kPa}$, Los resultados indican que la posición de carga tiene un efecto significativo en la respuesta mecánica del producto; éste resiste menos a la compresión lo cual puede ser originado a la heterogeneidad estructural de la pulpa-cáscara; Alvis et al. (2009) encontraron valores en ñame variedad Diamante 22, de $462.3 \mathrm{kPa}$ mientras que para el Pico de Botella es de $747.2 \mathrm{kPa}$, por lo tanto, para el caso de la batata donde su estructura es más compacta los valores son más altos. En las pruebas de penetración, de acuerdo a lo reportado por Buitrago et al. (2004), encontraron que la fuerza máxima promedio para romper la papa fue de $141,4 \pm 30.8 \mathrm{~N}$ lo cual se presenta una deformación de 18,9 $\mathrm{mm}$ después de atravesar el centro de la papa.

\section{CONCLUSIONES}

En las dos variedades de batata, los valores del esfuerzo máximo de penetración en el punto de $50 \%$ de la longitud del tubérculo no presenta diferencias significativas mientras que para el caso de los otros dos puntos de penetración 25 y $75 \%$ existen diferencias significativas entre las variedades de estudio; del mismo modo, el esfuerzo de fractura en flexión y en compresión es superior para la variedad amarilla (1262.85 Kpa y $0.753 \mathrm{Mpa}$ respectivamente) en relación con la variedad colorada (1212.17 Kpa y $0.772 \mathrm{Mpa}$ respectivamente). La variedad amarilla es más resistente a los esfuerzos aplicados para deformar el producto y por tanto, menos susceptible a daños causados en las etapas de cosecha, almacenamiento y manipulación.

\section{AGRADECIMIENTOS}

Los autores agradecen a la Universidad de Córdoba por el apoyo recibido para el desarrollo de la investigación.

\section{REFERENCIAS}

Alvarez, M.D. y W. Canet, Rheology characterization of fresh and cooked potato tissues (cv. Monalisa). Zeitschrift-fuer-Lebensmittel-Untersuchung-und-Forschung-A/Food-Research-and-Technology: 207(1), 5565 (1998)

Alvarez, M.D., M.J. Morillo y W. Canet, Characterization of the frying process of fresh and blanched potato strips using response surface methodology. European-Food-Research-and-Technology: 211(5), 326-335 (2000) 
Alvis, A., M. Páez y J. Lafont, Propiedades Mecánicas y Viscoelásticas del Name (Dioscórea alata). Inf. Tecnol. vol.20 (5) 75-81 (2009)

An, L. V., B. E. Frankow-Lindberg y J. E. Lindberg, Effect of harvesting interval and defoliation on yield and chemical composition of leaves, stems and tubers of sweet potato (Ipomoea batatas (Lam.)) plant parts. Field Crops Research, 82, 49-58 (2003)

Anzaldúa-Morales, A. y M.C. Bourne, Differences in texture and solids content of the cortex and pith tissues of potato tubers. Search: Agriculture: 37(1), 1-30 (1992)

Bruns, A.J. y M.C. Bourne, Effects of sample dimensions on the snapping force of crisp foods. Journal of Texture Studies: 6(4), 445-448 (1975)

Buitrago. G, P. Alonso, P. Alfonso y L. Osorno, Determinación de las características físicas y propiedades Mecánicas de papa cultivada en Colombia. Revista Brasileira de Engenharia Agrícola e Ambiental, 8(1), 102-110 (2004)

Ciro, H., L. Montoya, L. López y L. Millán, Caracterización de propiedades mecánicas del banano (Cavendish Valery), Rev. Fac. Nal. Agr. Medellín. 58(2) 2975-2988 (2005)

De Hombre, M.R. y J.A. Díaz-Abreu, Textura de Sólidos y Semisólidos. Capitulo 1.6. Libro Métodos para Medir Propiedades Físicas en Industria de Alimentos. Editores: J. de Dios Alvarado y J. M. Aguilera. Ed. Acribia, 109-134 (2001)

Farag, K. W., J. G. Lyng, D. J. Morgan y D. A. Cronin, Effect of low temperatures (18 to +5 degrees C) on the texture of beef lean. Meat Science, 81, 249-254 (2009)

Foegeding, E. A., E. Çakır y H. Koç, Using dairy ingredients to alter texture of foods: Implications based on oral processing considerations. International Dairy Journal, 20, 562-570 (2010)

Konopacka, D. y W. J. Plocharski, Effect of storage conditions on the relationship between apple firmness and texture acceptability. Postharvest Biology and Technology, 32, 205-211 (2004)

Lana, M. M., L. M. M. Tijskens y O. Van Kooten, Effects of storage temperature and fruit ripening on firmness of fresh cut tomatoes. Postharvest Biology and Technology, 35, 87-95 (2005)

Lima, I. y P. Singh, Mechanical properties of a fried crust. Journal of Texture Studies: 32(1), 31-40 (2001)

Martí, H., G. Corbino y H. Chludil, Batata: El redescubrimiento de un cultivo. Instituto Nacional de Tecnología Agropecuaria., vol.21 (121) 17-23 (2011)

Martí, H., Consuma más batata. Buenos Aires-Argentina, INTA, no. 17 (2012)

Mohsenim, N.N., Physical Properties of Agricultural Products. Transactions of the American Society of Agricultural Engineers: 8(1), 25-29 (1965)

Moreira, R.G, M.E Castell-Perez, y M.A. Barrufet; Deep-Fat Frying. Fundamentals and Applications. Aspen Publication, 88-101 (1999)

Morris, C. y G. A. Morris, The effect of inulin and fructo-oligosaccharide supplementation on the textural, rheological and sensory properties of bread and their role in weight management: A review. Food Chemistry, 133, 237-248 (2012)

Nwandikom, G.I., Yam tuber resistance of mechanical damage. Agricultural-Mechanization-in-Asia,Africa-and-Latin-America: 21(2), 33 -36 (1990)

Pitt, R.E., Stress-Strain and Failure Characteristics of Potato Tissue under cyclic loading. Journal of Texture Studies: 15, 131-155 (1984)

Qian Li, Li. Dong, Wang, Li-jun, Necati Özkan y Mao Zhi-huai. Dynamic viscoelastic properties of sweet potato studied by dynamic mechanical analyzer. Carbohydrate Polymers 79, 520-525 (2010) 
Saeleaw, M. y G. Schleining, A review: Crispness in dry foods and quality measurements based on acousticmechanical destructive techniques. Journal of Food Engineering, 105, 387-399 (2011b)

Teow, C. C., V. D. Truong, R. F. McFeeters, R. L. Thompson, K. V. Pecota y G. C. Yencho, Antioxidant activities, phenolic and $b$-carotene contents of sweet potato genotypes with varying flesh colours. Food Chemistry, 103, 829-838 (2007)

Tian, C. Y. y G. L. Wang, Study on the anti-tumor effect of polysaccharides from sweet potato. Journal of Biotechnology, 136, S351(2008)

Varela, P., A. Salvador y S. Fiszman; Changes in apple tissue with storage time rheological, textural and microstructural analyses. Journal of Food Engineering: 78(2), 622-629 (2007)

Voisey, P.W., N.W. Tape, y M. Kloek; Physical Properties of the Potato Tuber. Canadian Institute of Food Technology Journal: 2(2), 98-103 (1969)

Zhang, Z. T., C. W. Christopher y C. Harold, Biochemical changes during storage of sweet potato roots differing in dry matter content. Postharvest Biology and Technology, 24, 317-325 (2002) 